IOS Press

\title{
GR silencing impedes the progression of castration-resistant prostate cancer through the JAG1/NOTCH2 pathway via up-regulation of microRNA-143-3p
}

\author{
Linshen Zhang ${ }^{\mathrm{a}, \mathrm{b}}$, Hongjun Jiang ${ }^{\mathrm{a}}$, Yufan Zhang ${ }^{\mathrm{a}}$, Chenrong Wang ${ }^{\mathrm{a}}$, Xixi Xia ${ }^{\mathrm{a}}$ and Yi Sun ${ }^{\mathrm{a}, *}$ \\ ${ }^{a}$ Department of Clinical Laboratory Medicine, The First People's Hospital of Yunnan Province, Kunming, Yunnan, \\ China \\ b2016 Innovation Class, Kunming Medical University, Kunming, Yunnan, China
}

\begin{abstract}
.
BACKGROUND: Despite notable progression from a therapeutic point of view, castration resistant prostate cancer (CRPC) remains a clinical significant stumbling block. The current study aimed to elucidate the functional role of the gene glucocorticoid receptor (GR) in CRPC, and identify the contributions of the GR gene in CRPC in connection with microRNA-143-3p (miR-1433p)/Jagged1 (JAG1)/NOTCH2.

METHODS: The expression of GR and miR-143-3p in CRPC tissues and cells as well as JAG1/NOTCH2 expression in CRPC tissues was initially determined by quantitative polymerase chain reaction and Western blot analyses. The relationship among GR, JAG1, NOTCH2 and miR-143-3p was subsequently verified using the dual-luciferase reporter gene assay. ChIP assay confirmed the binding of GR to miR-143-3p promoter. Gain- and loss-function approaches were applied to ascertain the role of GR and miR-143-3p in progression of CRPC. Additionally, xenograft tumor models in nude mice were established to further confirm our results.

RESULTS: GR was found to be highly expressed while miR-143-3p was lowly expressed in the CRPC tissues and cells. Silencing GR reduced migration, invasion, proliferation and increased apoptosis of CRPC cells. GR was enriched in the miR-143-3p promoter region and could down-regulate miR-143-3p expression. The overexpression of miR-143-3p led to a reduction in the migration, invasion, proliferation and increased apoptosis of CRPC cells. JAG1 and NOTCH2 were the target genes of miR-143-3p, and GR up-regulated the JAG1/NOTCH2 expression by down-regulating miR-143-3p. Silencing JAG1/NOTCH2 inhibited epithelial-mesenchymal transition and CRPC progression in vitro. Furthermore, the in vitro findings were reproduced in the in vivo experiments.

CONCLUSION: The key findings of the current study demonstrated that silencing GR suppressed the progression of CRPC through the JAG1/NOTCH2 pathway via up-regulation of miR-143-3p.
\end{abstract}

Keywords: Glucocorticoid receptor, microRNA-143-3p, JAG1, NOTCH2, castration-resistant prostate cancer, migration, invasion

\footnotetext{
${ }^{*}$ Corresponding author: Yi Sun, Department of Clinical Laboratory Medicine, The First People's Hospital of Yunnan Province, No. 157, Jinbi Road, Kunming, Yunnan, 650031, China. Tel./Fax.: +86 871 63638431; E-mail: huludeng@126.com.
}

\section{Introduction}

Prostate cancer is widely regarded as the foremost malignancy afflicting males worldwide, with studies indicating nearly 1.3 million new cases and 359,000 related deaths worldwide in 2018 [1,2]. Androgen deprivation therapy (ADT) is a commonly used treatment method for symptomatic prostate cancer, often resulting

ISSN 1574-0153/20/\$35.00 (C) 2020 - IOS Press and the authors. All rights reserved

This article is published online with Open Access and distributed under the terms of the Creative Commons Attribution Non-Commercial License (CC BY-NC 4.0). 
in reduced levels of prostate-specific antigen [3]. Although most patients suffering from metastatic prostate cancer can benefit from ADT, almost all patients will develop castration-resistant prostate cancer (CRPC) approximately 18 to 24 months on average [4]. CRPC, formerly known as hormone-resistant prostate cancer, is now regarded as a form of progressive disease in spite of medical or surgical castration [5]. Commendable achievements have been made in relation to the drug treatment of CRPC, including sipuleucel-T, abiraterone acetate, and cabazitaxel, but the poor prognosis of patients with CRPC is still a major treatment challenge [6,7].

Glucocorticoid receptor (GR) represents a member of the superfamily of nuclear receptor transcription factors and has anti-proliferative and anti-apoptotic functions [8]. Previous research has highlighted increased expression and activity of GR in the progression of CRPC following androgen blockade [9]. Interestingly, investigation of dysregulated microRNAs (miRNAs) in CRPC has provided the possibility of new prognostic models for improving treatment success [10]. The crucial roles of miRNAs in cell proliferation and differentiation through modulation of the expression of specific genes have been demonstrated [11]. Recent literature has demonstrated that miR-143 suppresses prostate cancer cell proliferation, and migration, yielding a potential novel candidate for the treatment of prostate cancer [12]. Moreover, miR-143-3p has also been confirmed to exert anti-tumor effects in a variety of cancers, such as breast cancer and ovarian cancer $[13,14]$. Although miR-143-3p has been identified as a downregulated miRNA in metastatic prostate cancer [15], the role and mechanism by which miR-143-3p acted to affect cancer progression in this malignancy remains to be defined.

The application of a dual-luciferase reporter gene assay in our study suggested that Jagged1 (JAG1) and NOTCH2 were the targets of miR-143-3p. Notch signaling pathway plays an indispensable role in tissue development, and its ability to modulate cell growth and development has been elucidated to be correlated with the development of certain cancers, including prostate cancer [16]. JAG1, a ligand of the Notch signaling pathway, has also been reported to be associated with prostate cancer in a positive manner [17]. Following the aforementioned exploration of literature, the current study aimed to further elucidate the functional role of the gene GR in CRPC in connection with miR-1433p/JAG1/NOTCH2.

\section{Materials and methods}

\subsection{Ethics statement}

All samples were donated with the informed consent of patients, and the present study was conducted in accordance with the Declaration of Helsinki. The study protocol was approved by the Ethics Committee of the First People's Hospital of Yunnan Province. Animal use and experimental procedures were performed with the approval of the Experimental Animal Ethics Committee of the First People's Hospital of Yunnan Province. All efforts were made to reduce the suffering of animals.

\subsection{Sample collection}

A total of 63 patients with prostate cancer hospitalized at the First People's Hospital of Yunnan Province between 2016 and 2018 were recruited for sample collection. There were 32 patients with CRPC and 31 patients with androgen dependent prostate cancer (ADPC). Each specimen was histologically identified by prostate specific antigen staining [18].

\subsection{CRPC cell culture and transfection}

Human prostate cancer cell line LNCaP purchased from BNCC cell bank was cultured in Eagle's Mem Ebss Minimum Essential Medium containing 10\% fetal bovine serum (FBS) and $1 \%$ penicillin/streptomycin at $37^{\circ} \mathrm{C}$ with $5 \% \mathrm{CO}_{2}$ under $95 \%$ saturated humidity in an incubator. The solutions were renewed 2-3 times every week, while the cells in the logarithmic growth period were employed for subsequent experiments. The LNCaP cells were supplemented with the enzalutamide (Selleck Chemicals, Houston, TX, USA) for 2 h treatment. After the culture medium was removed, the cells were rinsed three times with phosphate buffer saline (PBS), detached with trypsin, and subsequently centrifuged at $1000 \mathrm{rpm}$ for $5 \mathrm{~min}$ at ambient temperature followed by removal of the supernatant. The culture medium was then added into cells for further culturing. After $24 \mathrm{~h}$, a large number of cells died, the dead cells were removed, after which the living cells were expanded. When cell density reached $80 \%-90 \%$, subculture was performed for 3 passages. The aforementioned methods were repeated twice (drug induction for 3 times, 4 weeks per period). The time of drug induction was increased to $4 \mathrm{~h}$, and the period was subsequently shortened to 10 days each time, after which the procedure was repeated. The duration of the entire 
Table 1

Primer sequences for RT-qPCR

\begin{tabular}{ll}
\hline \multicolumn{1}{c}{ Gene } & \multicolumn{1}{c}{ Sequences } \\
\hline GR (human) & F: 5'-CTGTCGCTTCTCAATCAGACTC-3' \\
& R: 5'-CCCAGGTCATTTCCCATCACTT-3' \\
miR-143-3p (human) & RT: 5'-GTCGTATCCAGTGCGTGTCGTGGAGTCGGCAATTGCACTGGATACGACTGAGCTA-3' \\
& F: 5'-AGTGCGTGTCGTGGAGTC-3' \\
& R: 5'-GCCTGAGATGAAGCACTGT-3' \\
& F: 5'-TCGGGTCAGTTCGAGTTGGA-3' \\
JAG1 (human) & R: 5'-CGTTCACGTTCTGCATGGAC-3' \\
& F: 5'-AATCCCTGACTCCAGAACG-3' \\
NOTCH2 (human) & R: 5'-TGGTAGACCAAGTCTGTGATGAT-3' \\
& F: 5'-CTCGCTTCGGCAGCACA-3' \\
U6 (human) & R: 5'-AACGCTTCACGAATTTGCGT-3', \\
& F: 5'-ACCCAGAAGACTGTGGATGG-3', \\
GAPDH (human) & R: 5'-TCTAGACGGCAGGTCAGGTC-3' \\
& RT: 5'-CTCAACTGGTGTCGTGGAGTCGGCAATTCAGTTGAGACCAGAGA-3' \\
miR-143-3p (mouse) & F: 5'-ACACTCCAGCTGGGGGTGCAGTGCTGCATC-3' \\
& R: 5'-TGGTGTCGTGGAGCCG-3' \\
& F: 5'-CTCGCTTCGGCAGCACA-3' \\
& R: 5'-GTCGTATCCAGTGCAGGTCCGAGGTATTCGCACTGGATACGACTCATCT-3'
\end{tabular}

Note: RT-qPCR, reverse transcription-quantitative polymerase chain reaction; F, forward; R, reverse; RT, reverse transcription; miR, microRNA; JAG1, Jagged 1; GAPDH, glyceraldehyde-3-phosphate dehydrogenase.

course lasted 6 months. Finally, enzalutamide-resistant cell line, Enz-LNCaP (or CRPC cells) was obtained.

Based on the sequences of GR, miR-143-3p, JAG1 and NOTCH2 known in National Center for Biotechnology Information, plasmids needed for the experiment were synthesized by Shanghai Sangon Biotech Co., Ltd., (Shanghai, China). Enz-LNCaP cells were transfected with the plasmids as follows: siRNA against GR (si-GR), GR overexpression plasmid (oe-GR), miR143-3p mimic, miR-143-3p-inhibitor, siRNA against JAG1 (si-JAG1), and siRNA against NOTCH2 (si$\mathrm{NOTCH} 2$ ) as well as their corresponding negative controls (NCs). One day prior to transfection, the cells at passage 3 exhibiting logarithmic growth were seeded into a 6-well plate and transfected $12 \mathrm{~h}$ later based on the instructions of the Lipofectamine 2000 (Invitrogen Inc., Carlsbad, CA, USA).

\subsection{RNA isolation and quantification}

Total RNA was extracted from tissues or cells collected $24 \mathrm{~h}$ after transfection by TRIZOL (Invitrogen). GR, miR-143-3p, JAG1 and NOTCH2 primers were designed and further synthesized by Invitrogen. Different reverse transcription (RT) kits, TaqMan ${ }^{\mathrm{TM}}$ MicroRNA Reverse Transcription Kit (4366596, Thermo Fisher Scientific, Rockford, IL, USA) and High-Capacity cDNA Reverse Transcription Kit (4368813, Thermo Fisher Scientific), were employed to reversely transcribe the obtained total RNA into cDNA. Real time quantitative polymerase chain reaction (qPCR) experi- ments were performed on ABI7500 qPCR instrument (Thermo Fisher Scientific) using the SYBR ${ }^{\circledR}$ Premix Ex TaqTM (Tli RNaseH Plus) kit (RR820A, TaKaRa, Japan) with glyceraldehyde-3-phosphate dehydrogenase (GAPDH) and U6 regarded as the internal reference (Invitrogen). The primers used are listed in Table 1. PCR was performed on a real-time fluorescence qPCR instrument (ABI, Foster City, CA, USA). The final data obtained were analyzed using the $2^{-\Delta \Delta \mathrm{Ct}}$ method.

\subsection{Protein isolation and quantification}

The total protein of tissues or cells was extracted using a Radio immunoprecipitation assay kit (R0010, Beijing Solarbio Science \& Technology Co., Ltd., Beijing, China), with protein concentration measured using the bicinchoninic acid method protein assay kit (GBCBIO Technologies Inc., Guangdong, China). A total of $40 \mu \mathrm{g}$ protein was extracted from each sample, separated by $10 \%$ of sodium dodecyl sulfate polyacrylamide gel electrophoresis, and subsequently transferred onto a polyvinylidene fluoride membrane. The membrane was sealed by Tris-Buffered Saline Tween20 containing $5 \%$ bovine serum albumin (BSA) solution at ambient temperature, and probed with diluted rabbit primary antibodies (CST, Danvers, MA, USA) to GR (\#12041, 1: 1000, Rabbit), JAG1 (\#2620, 1: 1000, Rabbit), NOTCH2 (\#4530, 1: 1000, Rabbit), E-cadherin (\#3195, 1: 1000, Rabbit), N-cadherin (\#13116, 1: 1000, Rabbit), Vimentin (\#5741, 1: 1000, Rabbit), GAPDH 
(\#5174, 1: 1000, Rabbit), B-cell lymphoma-2 (Bcl-2) (\#3498, 1: 1000, Rabbit), Cyclin D1 (\#2922, 1: 1000, Rabbit), Cleaved poly ADP-ribose polymerase (PARP) (\#94885, 1: 1000, Rabbit), and Cleaved caspase-3 (\#9654, 1: 1000, Rabbit) for incubation overnight at $4^{\circ} \mathrm{C}$. Next, the membrane was re-probed using secondary antibody goat anti-rabbit immunoglobulin G (IgG) (ab150077, 1: 1000, Abcam, Cambridge, UK) at room temperature. The band was developed by enhanced chemiluminescence. Image $\mathbf{J}$ software was used to evaluate the gray value of each band, with the gray value ratio of the target protein to that of the loading control protein band calculated.

\subsection{Verification of relationship between JAG1/NOTCH2 and miR-143-3p}

The biological prediction website microRNA.org was used for analysis of the target gene of miR-143-3p and dual-luciferase reporter gene assay was conducted to verify whether JAG1/NOTCH2 was a direct target gene of miR-143-3p. The constructed JAG1/NOTCH2-wild type (WT) and JAG1/NOTCH2-mutant (MUT) were co-transfected with miR-143-3p mimic or mimic NC to HEK-293T cells (Shanghai BeiNuo Biotechnology Co., Ltd., Shanghai, China). After 48-h transfection, the cells were collected and lysed, and luciferase activity was determined by means of luciferase assay kit (K801-200, Biovision, Milpitas, CA, USA) using the glomax20/20 luminometer (Promega WI, USA).

The miR-143-3p promoter region was cloned into the Luciferase vector of pmirGLO (Promega) to construct the miR-143-3p Promoter WT (wild-type miR-143-3p Promoter plasmid), miR-143-3p Promoter mut1 (miR143-3p Promoter plasmid at mutation site 1) and miR143-3p Promoter mut2 (miR-143-3p Promoter plasmid at mutation site 2). The HEK-293T cells were seeded into a 24-well plate. After $24 \mathrm{~h}, 100 \mathrm{ng}$ oe-NC or $100 \mathrm{ng}$ oe-GR were co-transfected with $50 \mathrm{nmol} / \mathrm{L}$ miR-143-3p Promoter plasmid according to Lipofectamine 2000 kit (Invitrogen), and the luciferase activity was measured $48 \mathrm{~h}$ after transfection.

\subsection{Determination of GR enrichment in the miR-143-3p promoter}

Chromatin immunoprecipitation (ChIP) assay was applied for determination of GR enrichment in the miR143-3p promoter. After the cells had been fixed with $4 \%$ formaldehyde (the final concentration of formaldehyde was $1 \%$ ), the cells were sonicated, followed by the addition of rabbit anti human interference GR antibody (\#12041, 1: 50, Rabbit, CST), and Protein A Agarose/SaLmon Sperm DNA to precipitate GR antibody-GR-miR-143-3p promoter complex. The complex was eluted to remove some nonspecific combination. The enriched GR-miR-143-3p promoter complex was obtained after elution, and then de-crosslinked. The enriched miR-143-3p promoter fragment was purified for PCR analysis.

\subsection{Measurement of drug-resistant $50 \%$ inhibitory concentration (IC50)}

The drug-resistant cells and parental LNCaP cells at logarithmic growth stage were seeded into 96-well plates $\left(7 \times 10^{3}\right.$ cells per well) pre-added with $100 \mu \mathrm{L}$ of culture solution per well. Next, $24 \mathrm{~h}$ later, each well was supplemented with $100 \mu \mathrm{L}$ of enzalutamide $(0 \mu \mathrm{M}, 2.5 \mu \mathrm{M}, 5 \mu \mathrm{M}, 10 \mu \mathrm{M}, 20 \mu \mathrm{M}, 40 \mu \mathrm{M}, 80 \mu \mathrm{M}$, $160 \mu \mathrm{M}, 320 \mu \mathrm{M}$ ), with 6 parallel wells set for each concentration. In the control group, $100 \mu \mathrm{L}$ medium was added into each well. After $48 \mathrm{~h}, 10 \mu \mathrm{L}$ cell counting kit 8 (CCK8) solution (C0038, Beyotime Institute of Biotechnology, Shanghai, China) was added to each well and cultured for $2 \mathrm{~h}$. After the supernatant had been removed, the optical density value at the wavelength of $450 \mathrm{~nm}$ was measured using an enzyme-linked immunosorbent assay. The IC50 and drug resistance index (RI) of drug-resistant cells and parental cells were calculated. RI = drug-resistant cell IC50/parental cell IC50.

\subsection{Cell proliferation assessment}

The cell culture plate was supplemented with 5ethynyl-2'-deoxyuridine (EdU) solution for 2-h incubation, added with $4 \%$ paraformaldehyde (100 $\mu \mathrm{L} /$ well $)$ for incubation at ambient temperature for $30 \mathrm{~min}$ and supplemented with $2 \mathrm{mg} / \mathrm{mL}$ glycine for 5 -min incubation. The cells were then further incubated with $100 \mu \mathrm{L} /$ well osmotic agents (PBS containing $0.5 \%$ TritonX-100) for $10 \mathrm{~min}$, rinsed once with PBS and supplemented with $1 \times$ Apollo staining solution (100 $\mu \mathrm{L} /$ well) for incubation for $30 \mathrm{~min}$ in conditions void of light. Finally, $1 \times$ Hoechst 33342 reaction solution (100 $\mu \mathrm{L} /$ well) was applied to cells for $30 \mathrm{~min}$. Pictures were taken under a fluorescence microscope, and the cells with red-stained nuclei were considered as positive cells. The numbers of positive and negative cells in any 3 fields of view were counted under the microscope. EdU labeling rate $(\%)=$ number of positive 
cells/(number of positive cells + number of negative cells) $\times 100 \%[19]$.

Cell cycle distribution was analyzed by flow cytometric analysis using PI single staining $48 \mathrm{~h}$ posttransfection. The cells were fixed with $70 \%$ cold absolute alcohol at $4^{\circ} \mathrm{C}$ for $2 \mathrm{~h}$ and stored at $-20^{\circ} \mathrm{C}$. Propidium iodide (PI) was added to cells in the presence of RNase A at room temperature for 30-min incubation. The cell cycle distribution was identified by means of flow cytometer equipped with Cell Quest software (FACSCalibur, BD Biosciences, San Jose, CA, USA).

\subsection{Cell apoptosis assessment}

Cell apoptosis was evaluated by terminal-deoxynucleoitidyl transferase mediated nick end labeling (TUNEL) assay followed by flow cytometric analysis.

The cells were placed on the cover slides at $37^{\circ} \mathrm{C}$ for $24 \mathrm{~h}$, rinsed with PBS, fixed with $4 \%$ paraformaldehyde for $60 \mathrm{~min}$, and subsequently rinsed again with PBS. The cells were then supplemented with PBS containing $0.1 \%$ Triton X-100 for an ice bath for $2 \mathrm{~min}$, followed by the addition of PBS and TUNEL detection solution for incubation at $37^{\circ} \mathrm{C}$ in dark conditions for $60 \mathrm{~min}$. After three PBS washes, the slides were sealed with anti-fluorescence quenching solution and observed under a fluorescence microscope, OLYMPUS FV1000. The excitation wavelength ranged from 450 $\mathrm{nm}$ to $500 \mathrm{~nm}$, the emission wavelength ranged from $515 \mathrm{~nm}$ to $565 \mathrm{~nm}$, and the apoptotic cells were noted to emit green fluorescence. Five fields were randomly selected to determine the number of normal cells and positive cells, after which the apoptosis rate was calculated (mean number of positively stained nuclei/number of all nuclei $\times 100 \%$ ).

Flow cytometric analysis was further performed using an Annexin V-fluoresceine isothiocyanate (FITC)/PI dual staining kit at $48 \mathrm{~h}$ after transfection. Briefly, 10 $\times$ Binding Buffer was diluted to $1 \times$ Binding Buffer by deionized water. The cells were collected by centrifugation at $2000 \mathrm{rpm}$ for $5 \mathrm{~min}$ at room temperature and re-suspended in $1 \times$ PBS, followed by centrifugation at $200 \mathrm{rpm}$ for 5-10 min. The cells were then re-suspended in $300 \mu \mathrm{L} 1 \times$ Binding Buffer and incubated with $5 \mu \mathrm{L}$ Annexin V-FITC at room temperature for 15 min under condition void of light. Following 5 -min ice bath with $5 \mu \mathrm{L}$ PI under dark conditions, FITC signal was detected on a flow cytometer (Cube6, Partec, Franklin Park, IL, USA) at wavelengths of $480 \mathrm{~nm}$ and $530 \mathrm{~nm}$ and PI was detected at a wavelength $>575 \mathrm{~nm}$.

\subsection{Cell migration assessment}

The cells were seeded into a 6-well plate at a density of $2.5 \times 10^{4}$ cells $/ \mathrm{cm}^{2}$ and cultured for $24 \mathrm{~h}$. The medium was then removed, with scratches made using a sterilized Tip $(100 \mu \mathrm{L})$. After two PBS washes, SmGM-2 ${ }^{\mathrm{TM}}$ medium containing $5 \%$ FBS was added to the cells for further culture. Images of each well at $0 \mathrm{~h}$ and $24 \mathrm{~h}$ after the scratches had been made were captured using an inverted microscope, with 3 duplicated wells in each group. The width of each scratch was determined using Image $\mathbf{J}$ software, with the migration ability of the cells evaluated by comparing the scratch width of each group. Scratch healing rate $=$ (scratch width at $0 \mathrm{~h}-$ scratch width at $24 \mathrm{~h}$ )/scratch width at $0 \mathrm{~h}$ $\times 100 \%$.

\subsection{Cell invasion assessment}

After transfection for $24 \mathrm{~h}$, the cells were starved in serum-free medium for $24 \mathrm{~h}$, detached, rinsed twice with PBS and re-suspended with serum-free medium Opti-MEM (31985008, Nanjing SenBeiJia Biological Technology Co., Ltd., Nanjing, Jiangsu, China) containing $10 \mathrm{~g} / \mathrm{L} \mathrm{BSA}$ with the cell density adjusted to 3 $\times 10^{4}$ cells/mL. Matrigel (40111ES08, Shanghai Yeason Biotechnology Co., Ltd., Shanghai, China) diluted at a ratio of 1: 8 was used to cover the upper surface of the bottom membrane of Transwell chamber after which it was air-dried overnight at $4^{\circ} \mathrm{C}$. After routine detachment, the cells in each group were rinsed twice with PBS and re-suspended with Roswell Park Memorial Institute (RPMI) 1640 medium with the cell density adjusted to $1 \times 10^{5}$ cells/mL. Cell suspension $(200 \mu \mathrm{L})$ was added to the apical chamber of Transwell chamber with Matrigel (BD Biosciences), and the RPMI 1640 medium containing 20\% FBS (Gibco Company, Grand Island, NY, USA) was subsequently added to the basolateral chamber for incubation at $37^{\circ} \mathrm{C}$ with $5 \%$ $\mathrm{CO}_{2}$ for $24 \mathrm{~h}$. The Transwell chamber was removed followed by removal of the non-invasive cells using cotton swabs, with the remaining cells fixed with $4 \%$ paraformaldehyde for $15 \mathrm{~min}$, stained with $0.5 \%$ crystal violet solution (prepared with methanol) for $15 \mathrm{~min}$, and rinsed three times with PBS. Next, 5 visual fields were selected at random in order to observe and analyze the invasive cells using an inverted microscope (XDS800D; Shanghai Cai Kang Optical Instrument Co., Ltd., Shanghai, China). The number of invasive cells in each group was calculated with 3 duplicated wells set. Other reagents were purchased from Beyotime. 


\subsection{Xenograft tumor in nude mice}

Ten BALB/c nude mice (purchased from Shanghai Lingchang company, Shanghai, China) aged between 5 and 7 weeks, weighing about (18 to 22) g were selected and raised under a specific pathogen-free environment at the animal experiment center of the First People's Hospital of Yunnan Province. The mice were fed with sterile food and drinking water in a comfortable temperature environment under a 12-h day/night cycle. The mice were acclimatized for 7 days. The shRNA sequence was inserted into the shRNA fluorescence expression vector $\mathrm{pSIH} 1-\mathrm{H} 1$-copGFP for gene silencing, and sh-NC and shRNA against GR (sh-GR) were all constructed by GenePharma Co., Ltd. (Shanghai, China). The 293T cells were introduced for lentivirus packaging and cultured in a complete RPMI-1640 culture medium supplemented with $10 \%$ FBS and passaged every other day. The virus was collected after which the lentivirus $\left(1 \times 10^{8} \mathrm{TU} / \mathrm{mL}\right)$ was transduced into the cells. Enz-LNCaP cell lines with stably infected with sh-NC and sh-GR lentivirus were constructed and made into $5 \times 10^{6}$ cells $/ \mathrm{mL}$ cell suspension, respectively. Cell suspension $(0.2 \mathrm{~mL})$ was injected subcutaneously into the nude mice using a $1 \mathrm{~mL}$ syringe. Tumor formation was analyzed and recorded on a daily basis. After tumor growth became more pronounced, the long diameter and short diameter of the tumor were measured using vernier caliper every 5 days. After 40 days of feeding, the nude mice were euthanized, with their respective subcutaneous tumors obtained for RT-qPCR and Western blot analysis.

\subsection{Statistical analysis}

All data were processed by SPSS 21.0 statistical software (IBM Inc., Armonk, NY, USA). Measurement data were expressed as mean \pm standard deviation. Paired $t$-test was employed to compare the data of the two groups of paired designs that followed normal distribution and homogeneity of variance, while unpaired $t$-test was used to compare the data of two groups with unpaired designs that conformed to normal distribution and homogeneity of variance. Data comparison among multiple groups was analyzed by one-way analysis of variance (ANOVA), followed by Tukey's post hoc test. Data from different groups at different time points were analyzed by repeated measures ANOVA with Bonferroni post hoc test. Pearson correlation analysis was adopted to analyze the expression correlation between miR-143-3p and JAG1/NOTCH2. $p<0.05$ was considered to be indicative of statistically significant difference.

\section{Results}

\subsection{High expression of $G R$ in $C R P C$}

Changes of GR in CRPC were initially detected by RT-qPCR. The results obtained illustrated that the GR expression in CRPC tissues was significantly elevated than that in ADPC tissues $(p<0.05)$ (Fig. 1A). The IC50 of parental cell line $\mathrm{LNCaP}$ was $48.31 \mu \mathrm{M}$ and the IC50 of Enz-LNCaP cell line was $264.18 \mu \mathrm{M}$ as measured by CCK 8 assay, which indicated that the EnzLNCaP cell line with high drug resistance was constructed (Fig. 1B). The RT-qPCR results revealed that the GR expression in the Enz-LNCaP cells was significantly increased when compared to that of the $\mathrm{LNCaP}$ cells $(p<0.05)$ (Fig. 1C). Western blot analysis results demonstrated that the protein expression of GR was also markedly higher in the CRPC tissues when compared to the ADPC tissues and that was higher in the Enz-LNCaP cells than in parental LNCaP cells (Fig. 1D). Altogether, the above results indicated that GR was expressed at a high level in the CRPC tissues and cells.

\subsection{Down-regulation of GR curbed the proliferation, migration and invasion of CRPC cells}

The underlying mechanism by which the expression of GR influences the biological characteristics of CRPC cells was investigated. In order to further explore the function of GR in CRPC, the proliferation, cycle distribution, migration, invasion and apoptosis abilities of Enz-LNCaP cells were detected by EdU assay, PI single staining, wound healing assay, Transwell assay, TUNEL staining and Annexin V-FITC/PI double staining, respectively. The results revealed that in EnzLNCaP cells, GR silencing resulted in decreased proliferation, migration, diminished invasive abilities and restrained cell cycle progression while increasing the apoptosis of cells $(p<0.05)$ (Fig. 2A-D, Supplementary Fig. 1A and B). The above results demonstrated that GR silencing could promote cell apoptosis, while suppressing cancer progression in CRPC cells.

\subsection{GR promoted progression of CRPC via suppression of miR-143-3p}

Next, the relationship between GR and miR-143$3 p$ was assessed, in addition to exploring their effects on CRPC progression. The binding sites of GR and miR-143-3p promoter were predicted by the website 

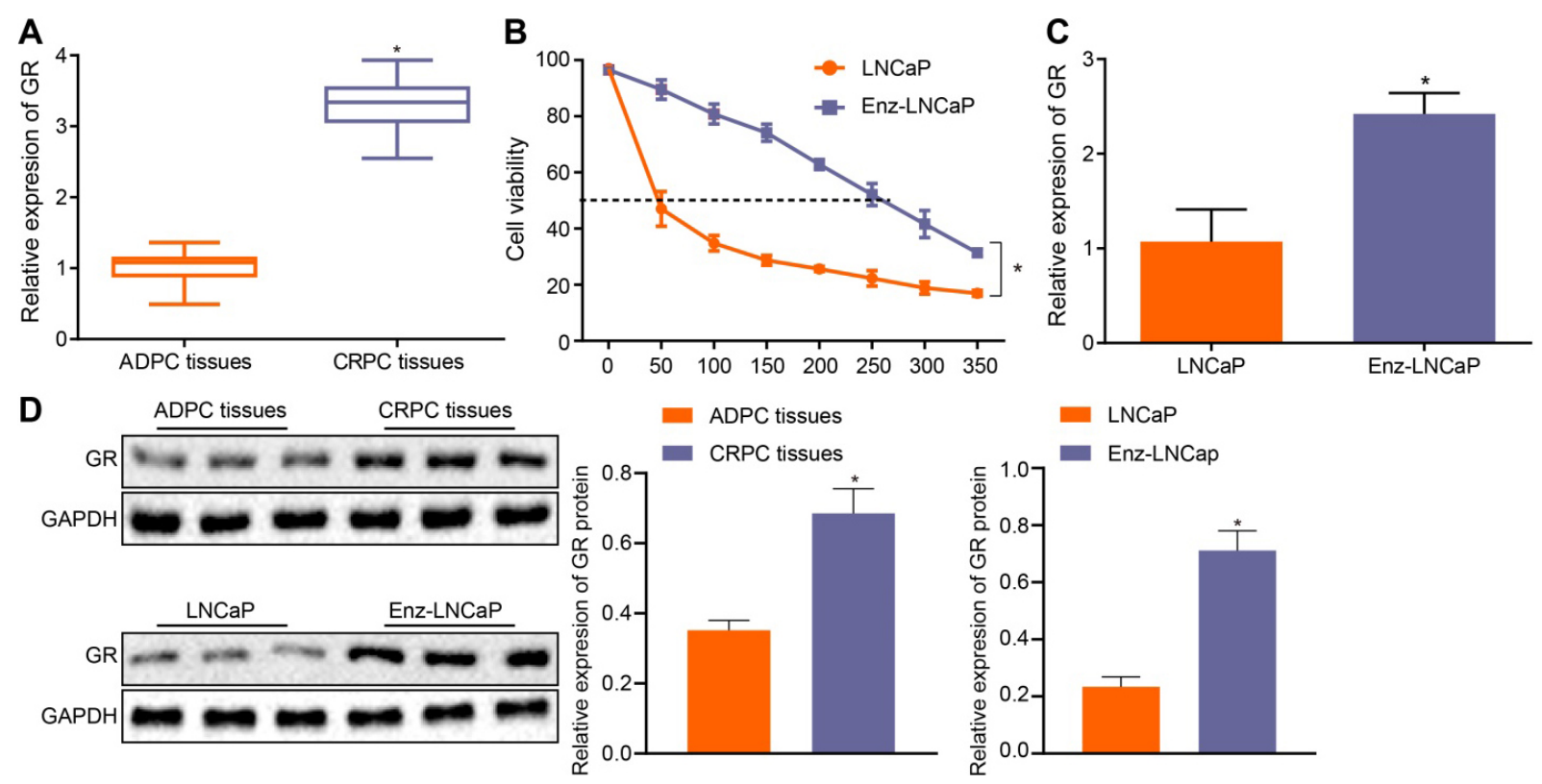

Fig. 1. GR exhibits a high expression in CRPC. A, The expression of GR in CRPC and ADPC tissues determined by RT-qPCR, ${ }^{*} p<0.05$ compared with ADPC tissues. B, The resistance of LNCaP cells to enzalutamide detected by CCK8 assay. C, The relative expression of GR in LNCaP and Enz-LNCaP cells determined by RT-qPCR. D, Grey value analysis and quantification of GR protein normalized to GAPDH in LNCaP and Enz-LNCaP cells as well as in the CRPC and ADPC tissues and LNCaP and Enz-LNCaP cells by Western blot analysis. ${ }^{*} p<0.05$ compared with ADPC tissues or LNCaP cells. All the measurement data were expressed as mean \pm standard deviation. Paired $t$-test $(n=8)$ was used to compare the paired data, and the unpaired $t$-test was used to compare the unpaired data that followed normal distribution and homogeneity of variance. Data of different groups at different time points were compared by repeated measures ANOVA with Bonferroni post hoc test. The cell experiment was repeated three times independently.

A

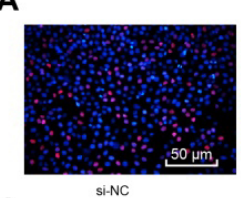

C

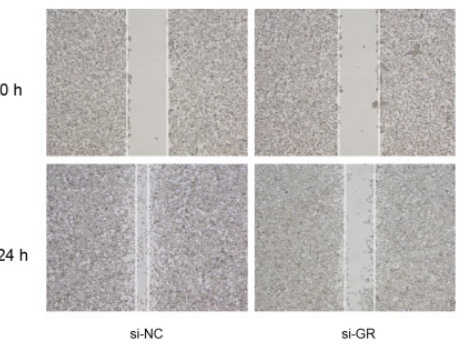

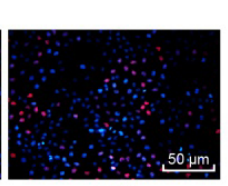

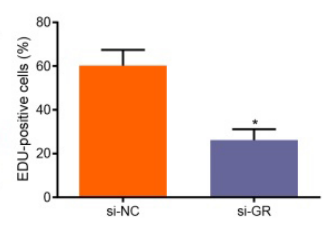

D

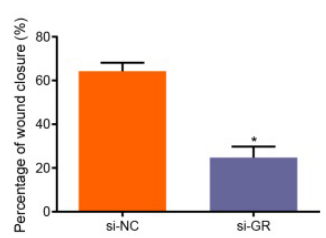

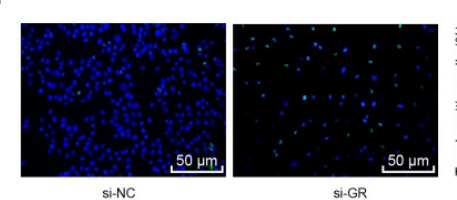

si-GR
si-NC
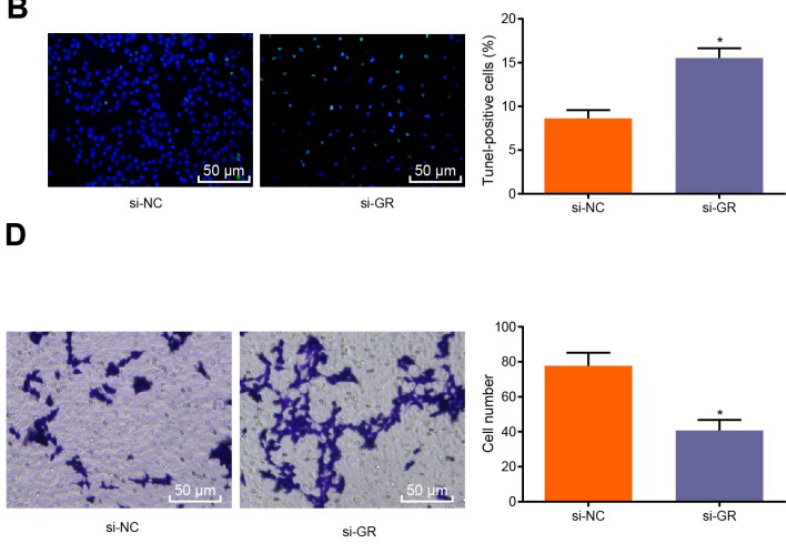

si-NC

(1)

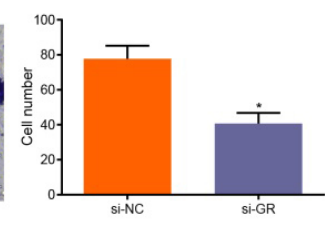

Fig. 2. Down-regulation of GR inhibits the malignant biological characteristics of CRPC cells. A, EdU assay was used to detect the proliferation of cells $(200 \times$, scale: $50 \mu \mathrm{m})$. B, TUNEL assay was used to detect cell apoptosis $(200 \times$, scale: $50 \mu \mathrm{m})$. C, The wound healing assay was designed to detect cell migration. D, Transwell assay was applied to measure the cell invasion $(200 \times$, scale: $50 \mu \mathrm{m}) .{ }^{*} p<0.05$ compared with cells treated with si-NC. All the measurement data were expressed as mean \pm standard deviation. The unpaired $t$-test was used to compare the data of the two groups of unpaired designs that followed normal distribution and homogeneity of variance. The cell experiment was repeated three times independently.

(https:/www.ncbi.nlm.nih.gov/genome/gdv/browser/? context $=$ GEO\&acc $=$ GSE91365). A specific binding site between the GR gene sequence and the miR-143-3p promoter sequence was identified, suggesting that GR might be related to the miR-143-3p promoter (Fig. 3A). The binding of GR to miR-143-3p promoter was further 

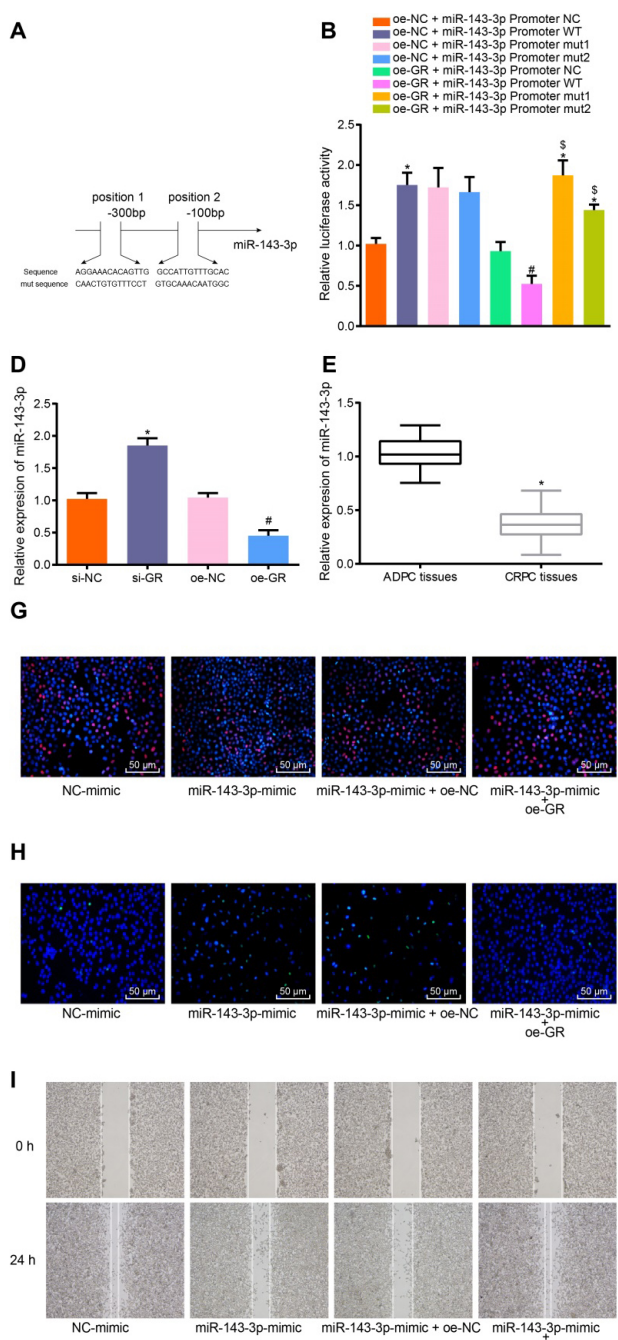

c
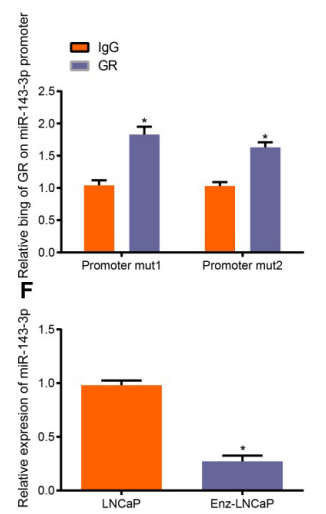

NC-mimic

3p-mimic

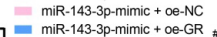

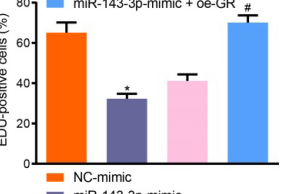

NC-mimic

miR-143-3p-mimic

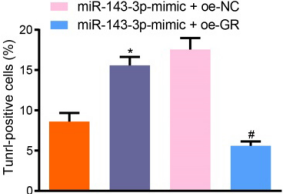

- NC-mimic

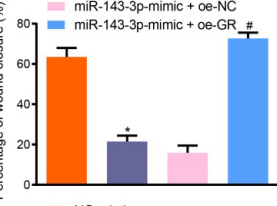

= NC-mimic
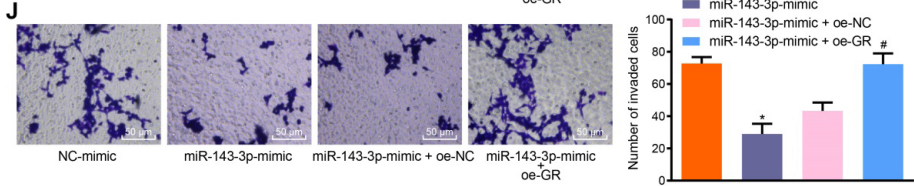

Fig. 3. GR promotes the progression of CRPC in vitro via suppression of miR-143-3p. A, The prediction of GR binding sites in miR-143-3p promoter and the mutation sequence produced by site mutations. B, The binding relationship between miR-143-3p and GR analyzed by dual-luciferase reporter assay, ${ }^{*} p<0.05$ compared with the oe-NC + miR-143-3p Promoter NC group; $\# p<0.05$ compared with the oe-GR + miR-143-3p Promoter NC group; ${ }^{\$} p<0.05$ compared with the oe-GR + miR-143-3p Promoter WT group. C, The enrichment of GR in miR-143-3p promoter detected by ChIP assay. ${ }^{*} p<0.05$ compared with the IgG group. D, The expression of miR-143-3p after GR silencing or overexpression determined by RT-qPCR, ${ }^{*} p<0.05$ compared with cells treated with si-NC; ${ }^{\#} p<0.05$ compared with cells treated with oe-NC. E, The expression of miR-143-3p in CRPC and ADPC tissues determined by RT-qPCR, ${ }^{*} p<0.05$ compared with ADPC tissues. F. The expression of miR-143-3p in LNCaP and Enz-LNCaP cells determined by RT-qPCR, ${ }^{*} p<0.05$ compared with LNCaP cells. G, Detection of cell proliferation by EdU assay, $(200 \times$, scale $50 \mu \mathrm{m}),{ }^{*} p<0.05$ compared with cells treated with NC-mimic; ${ }^{\#} p<0.05$ compared with cells treated with miR-143-3p mimic and oe-NC. H, Detection of apoptosis by TUNEL assay $(200 \times$, scale $50 \mu \mathrm{m}) .{ }^{*} p<0.05$ compared with the NC-mimic group, ${ }^{\#} p<0.05$ compared with the miR-143-3p mimic + oe-NC group. I, The cell migration ability detected by wound healing assay. ${ }^{*} p<0.05$ compared with the NC-mimic group; ${ }^{\#} p<0.05$ compared with the miR-143-3p mimic + oe-NC group. J, The invasive ability of cells detected by Transwell assay $(200 \times$, scale $50 \mu \mathrm{m}),{ }^{*} p<0.05$ compared with the NC-mimic group, $\# p<0.05$ compared with the miR-143-3p-mimic + oe-NC group. Measurement data were expressed as mean \pm standard deviation. Paired $t$-test $(n=8)$ was used to compare the data of the two groups of paired designs that followed normal distribution and homogeneity of variance, and the unpaired $t$-test was used to compare the data of the two groups of unpaired designs that followed normal distribution and homogeneity of variance. Data among multiple groups were analyzed by one-way ANOVA, followed by Tukey's post hoc test. The cell experiment was repeated three times independently. 
A
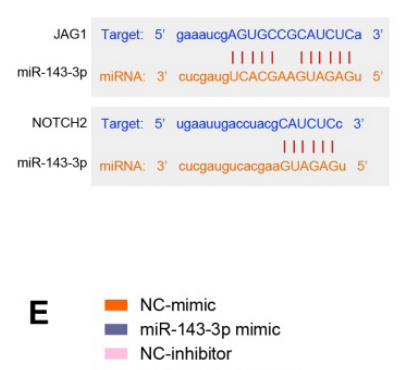

miR-143-3p-inhibito

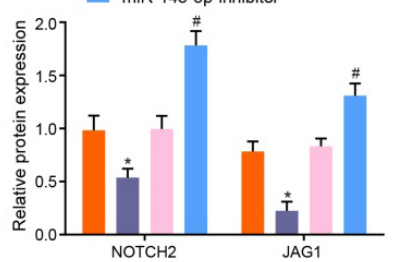

I

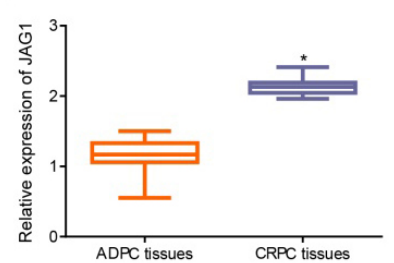

B

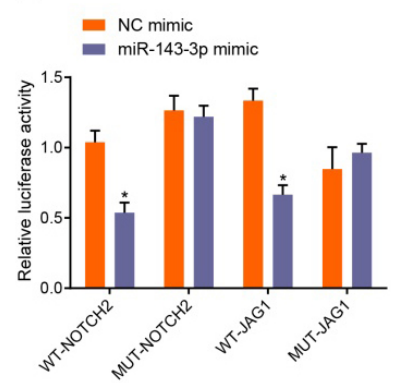

$F=0 \begin{array}{ll}\text { oe-NC } \\ \text { oe-GR }\end{array}$

sinc

- si-GR
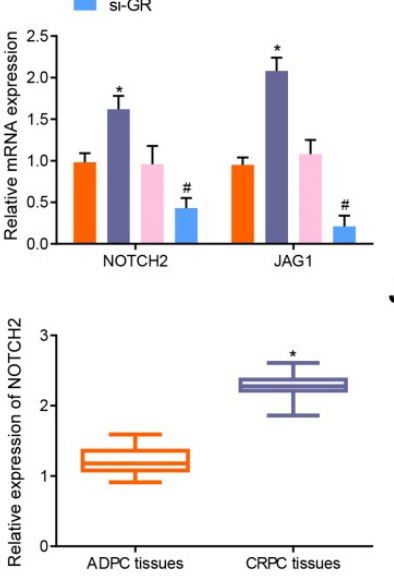

C $=$ NC-mimic

- miR-143-3p mimic

NC-inhibitor

- miR-143-3p-inhibitor

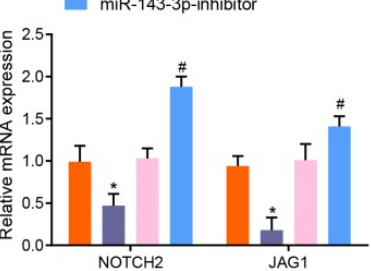

G
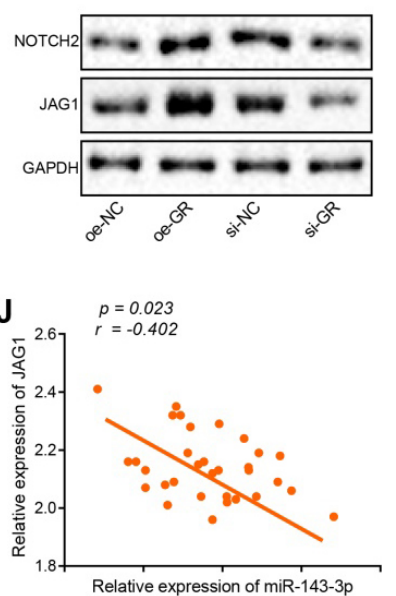

D

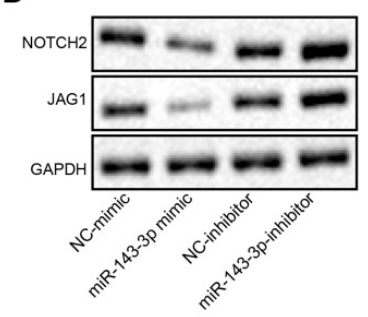

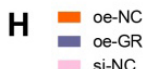

si-NC
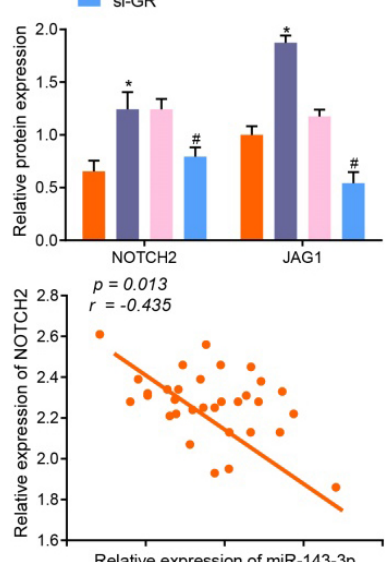

Fig. 4. GR modulates the expression of the target gene JAG1/NOTCH2 of miR-143-3p. A, The binding sites of miR-143-3p and JAG1/NOTCH2 predicted by websites. B, Identification of binding relationship between miR-143-3p and JAG1/NOTCH2 by dual-luciferase reporter assay, ${ }^{*} p<0.05$ compared with the wild type NC group. C, Determination of JAG1/NOTCH2 mRNA expression after miR-143-3p mimic or inhibitor transfection by RT-qPCR, ${ }^{*} p<0.05$ compared with cells treated with NC-mimic, ${ }^{\#} p<0.05$ compared with cells treated with NC-inhibitor. $\mathrm{D}$, Grey value analysis and quantification of JAG1/NOTCH2 protein expression after miR-143-3p mimic or inhibitor transfection normalized to GAPDH by Western blot analysis, ${ }^{*} p<0.05$ compared with cells treated with NC-mimic, ${ }^{*} p<0.05$ compared with cells treated with NC-inhibitor. E, The mRNA expression of JAG1/NOTCH2 after treatment of oe-GR or si-GR detected by RT-qPCR, ${ }^{*} p<0.05$ compared with cells treated with oe-NC group, ${ }^{\#} p<0.05$ compared with cells treated with si-NC. F, Grey value analysis and quantification of JAG1/NOTCH2 protein following treatment with oe-GR or si-GR normalized to GAPDH by Western blot analysis. ${ }^{*} p<0.05$ compared with cells treated with oe-NC, ${ }^{\#} p<0.05$ compared with cells treated with si-NC. G, Expression of JAG1/NOTCH2 in the CRPC and ADPC tissues determined by RT-qPCR, ${ }^{*} p<0.05$ compared with ADPC tissues. H, Pearson correlation was used to analyze the relationship of miR-143-3p and JAG1 $(p=$ 0.023, $r=-0.402) / \mathrm{NOTCH} 2(p=0.013, r=-0.435)$ in CRPC. In panels B, C, D, E, F, the measurement data were expressed as mean \pm standard deviation. The paired $t$-test $(n=8)$ was used to compare the data of the two groups of paired designs that followed normal distribution and homogeneity of variance, and the unpaired $t$-test was employed to compare the data of the two groups of unpaired designs that followed normal distribution and homogeneity of variance. Data comparison among multiple groups was analyzed by one-way ANOVA, followed by Tukey's post hoc test. The cell experiment was repeated three times independently.

verified by the findings of the dual-luciferase reporter assay (Fig. 3B), with the results showing that in comparison with the oe-GR + miR-143-3p Promoter WT group, the luciferase signal was increased in the oeGR + miR-143-3p Promoter mut1 transfection group and oe-GR + miR-143-3p Promoter mut 2 transfection group $(p<0.05)$. ChIP test results demonstrated significantly elevated GR enrichment in the GR group relative to $\operatorname{IgG}(p<0.05)$ (Fig. 3C). RT-qPCR results indicated that silencing GR up-regulated the expression of miR-143-3p in CRPC cells, while overexpressed GR decreased the expression of miR-143-3p $(p<0.05)$ (Fig. 3D). The results above elucidated that overexpressed GR was enriched in the promoter region of miR-143-3p and the miR-143-3p expression was therefore inhibited. RT-qPCR was carried out to determine the expression of miR-143-3p in CRPC and ADPC tissues, with the results found that the miR-143-3p expression in CRPC tissues was significantly diminished than that in ADPC tissues $(p<0.05)$ (Fig. 3E). Besides, lower miR-143-3p expression was also observed in EnzLNCaP cells versus in parental LNCaP cells (Fig. 3F). 
Subsequently, effects of miR-143-3p and GR on CRPC cell biological characteristics were analyzed by EdU assay, TUNEL staining, wound healing and Transwell assay. The results showed that Enz-LNCaP cells treated with miR-143-3p mimic presented decreased proliferation (Fig. 3G), migration (Fig. 3I) and invasion (Fig. 3J) abilities as well as increased apoptosis ability (Fig. 3H); while the Enz-LNCaP cells treated with overexpressed GR showed reversed changes in those characteristics (Fig. 3G-J) $(p<0.05)$. The above results implied that GR promoted the progression of CRPC by inhibiting miR-143-3p.

\subsection{GR regulated the expression of the target gene JAG1/NOTCH2 of miR-143-3p}

Next, the relationship between GR, miR-143-3p and JAG1/NOTCH2 was evaluated. The binding site of miR-143-3p and JAG1/NOTCH2 was predicted on the biological prediction website (Fig. 4A), after which dual-luciferase reporter gene assay was employed to verify whether JAG1/NOTCH2 was the target of miR143-3p (Fig. 4B), with the experimental results indicating that when compared with the NC mimic group, the luciferase signal of the miR-143-3p/WT-JAG1 or miR-143-3p/WT-NOTCH2 co-transfection group decreased $(p<0.05)$. However, no significant difference was detected in relation to the luciferase activity of mutant 3'UTR $(p>0.05)$. Cells transfected with miR-143 mimic had reduced JAG1/NOTCH2 mRNA and protein expression, on the contrary, cells transfected with miR143 inhibitor had increased JAG1/NOTCH2 mRNA and protein expression ( $p<0.05$, Fig. $4 \mathrm{C}$ and D). Furthermore, delivery of oe-GR was observed to elevate the expression of JAG1/NOTCH2, while transfection of siGR could decrease the expression of JAG1/NOTCH2 $(p<0.05)$ (Fig. 4E and F). RT-qPCR indicated that JAG1/NOTCH2 was notably increased in the CRPC tissues $(p<0.05)$ (Fig. 4G). The results of correlation analysis (Fig. $4 \mathrm{H}$ ) revealed the existence of an inverse relationship between miR-143-3p and JAG1/NOTCH2 expression in CRPC. These results provided evidence proving that GR modulated the expression of miR-143$3 p$ to further mediate its target gene JAG1/NOTCH2.

\subsection{GR/miR-143-3p/JAG1/NOTCH2 axis was involved in progression of CRPC}

It is speculated that GR may affect CRPC growth and metastasis through JAG1/NOTCH2 by mediating the expression of miR-143-3p. The RT-qPCR and West- ern blot analysis results revealed that the expression of JAG1/NOTCH2 was markedly elevated in Enz-LNCaP cells than in parental LNCaP cells $(p<0.05)$ (Fig. 5A and $\mathrm{B})$. The proliferative, apoptotic, migrating and invading abilities of Enz-LNCaP cells were evaluated by EdU assay, TUNEL staining, wound healing assay, and Transwell assay, respectively. The results demonstrated that silencing JAG1 or silencing NOTCH2 inhibited Enz-LNCaP cell proliferation, migration and invasion while promoting Enz-LNCaP cell apoptosis. Besides, overexpression of GR could abolish the effect of silencing JAG1 or silencing NOTCH2 on the aforementioned cell biological characteristics (Fig. 5C-F). Furthermore, expression of epithelial-mesenchymal transition (EMT)-related proteins after transfection with si-JAG1, si-NOTCH2, and oe-GR was analyzed by Western blot analysis. The results further implied that silencing JAG1 or NOTCH2 contributed to higher E-cadherin expression along with lower $\mathrm{N}$-cadherin/Vimentin expression $(p<0.05)$. Additionally, the cells transfected with siJAG1 and oe-GR had decreased E-cadherin expression while increased $\mathrm{N}$-cadherin/Vimentin expression than the cells transfected with si-JAG1 and oe-NC $(p<$ $0.05)$. The cells transfected with si-NOTCH2 and oeGR had decreased E-cadherin expression while elevated $\mathrm{N}$-cadherin/Vimentin expression than cells transfected with si-NOTCH2 and oe-NC $(p<0.05)$ (Fig. 5G). Altogether, the GR/miR-143-3p/JAG1/NOTCH2 axis affected the biological characteristics of Enz-LNCaP cells through mediating EMT.

\subsection{GR/miR-143-3p/JAG1/NOTCH2 axis regulated tumorigenesis of CRPC cells in vivo}

The xenograft tumor model in nude mice was established in order to further explore the role of GR/miR143-3p/JAG1/NOTCH2 in CRPC in vivo. The results collected revealed that following injection of cells infected with lentivirus expressing sh-GR, the subcutaneous tumor volume (Fig. 6A), tumor growth rate (Fig. 6B), and the final tumor weight (Fig. 6C) were notably decreased $(p<0.05)$. The mice injected with cells infected with lentivirus expressing sh-GR displayed increased miR-143-3p, Cleaved-PARP, Cleaved-Caspase 3 and E-cadherin expression, and decreased expression of GR, Bcl-2, Cyclin D1, JAG1, NOTCH2, N-cadherin and Vimentin $(p<0.05)$ (Fig. 6D and E). Altogether, the above results demonstrated that GR silencing in vivo could exert inhibitory effects on cell proliferation and EMT, and promotive effect on cell apoptosis, ultimately slowing down the tumorigenicity of the CRPC cells in mice via JAG1/NOTCH2 by up-regulating miR-143-3p. 


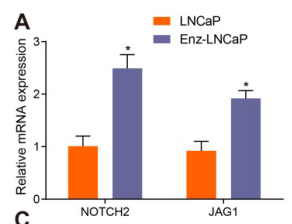

B
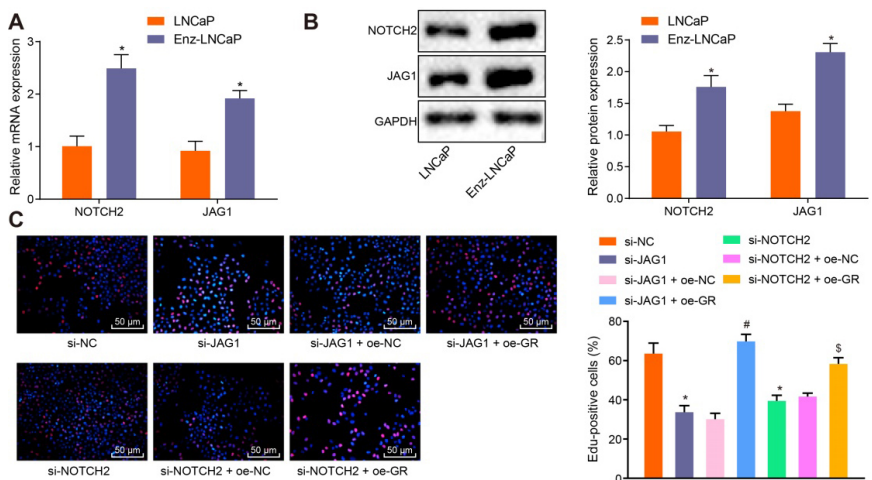

Si-AAG1 + oe-GR
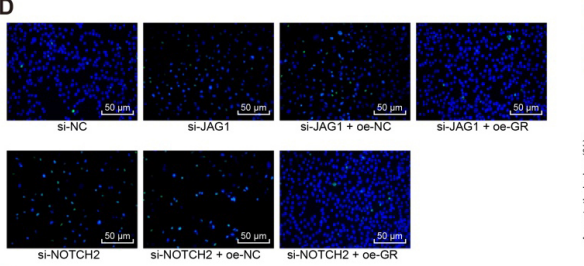

$=$ si-NC = si-NOTCH2

siJAG1
siJAG1 +oe-NC - siJAGI + oe-Gr
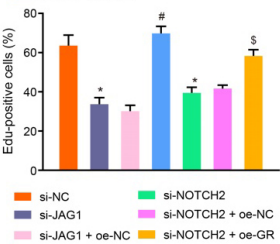

- siJAG1 + oe-GR
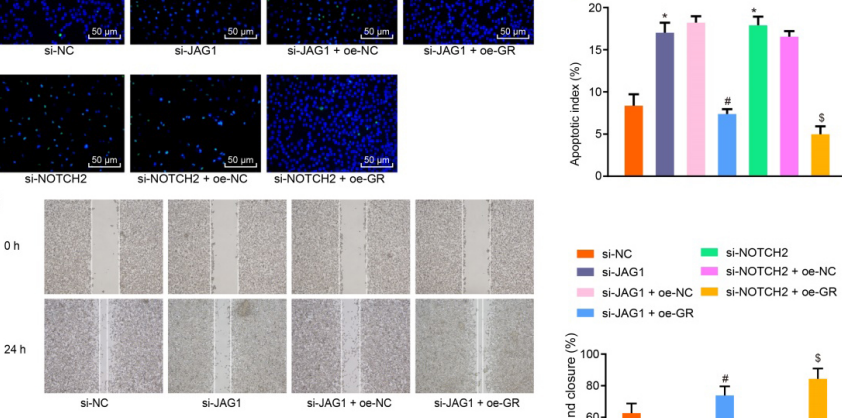

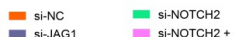

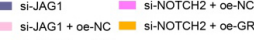
= Si-JAG1 + oe-GR
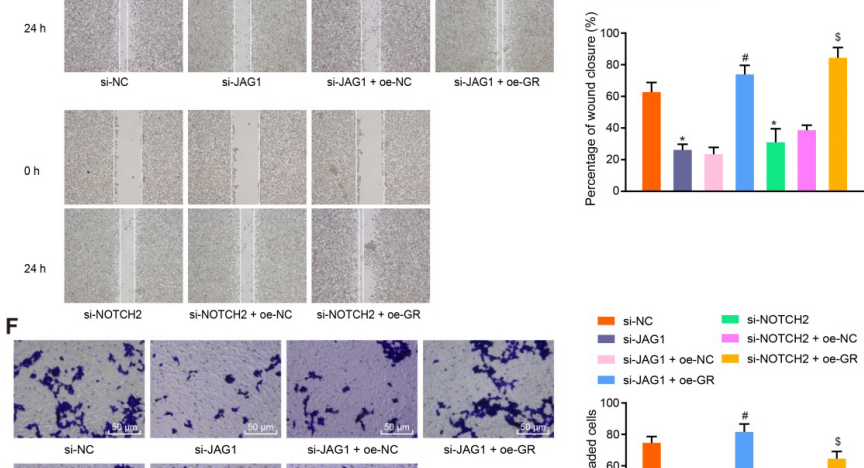

E si-NC $=$ siNOTCH2

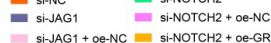
SiJAG1+ oe-NC = si-JAG1 + oe-GR
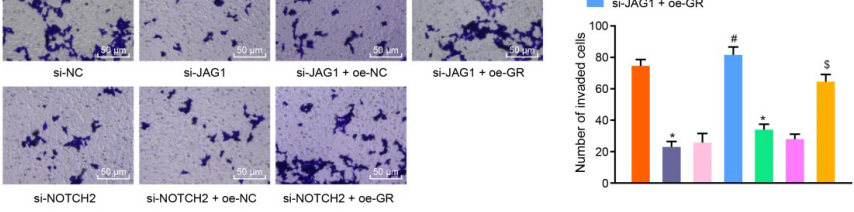

StNOTCH2

SiNOTCH2 + OQ-NC

G

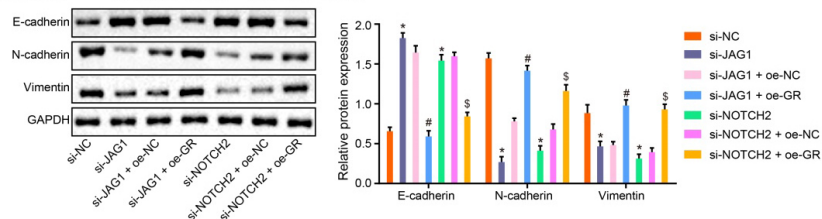

Fig. 5. GR/miR-143-3p/JAG1/NOTCH2 axis involves in the progression of CRPC. A, The expression of JAG1/NOTCH2 in LNCaP and Enz-LNCaP cells determined by RT-qPCR, ${ }^{*} p<0.05$ compared with LNCaP cells. B, Grey value analysis and quantification of JAG1/NOTCH2 protein normalized to GAPDH in LNCaP and Enz-LNCaP cells by Western blot analysis, ${ }^{*} p<0.05$ compared with LNCaP cells. C, EdU assay was applied for detecting cell proliferation $(200 \times$, scale: $50 \mu \mathrm{m})$. D, TUNEL assay was conducted for detecting cell apoptosis $(200 \times$, scale: $50 \mu \mathrm{m})$. E, Detection of cell migration ability by wound healing assay. F, Detection of cell invasion ability by Transwell assay ( $200 \times$, scale: $50 \mu \mathrm{m})$. $\mathrm{G}$, Western blot analysis was performed to detect the protein expression of JAG1, NOTCH2, E-cadherin, N-cadherin and Vimentin normalized to GAPDH. In panel C, D, E, F, G, ${ }^{*} p<0.05$ compared with cells treated with si-NC, ${ }^{*} p<0.05$ compared with cells treated with si-JAG1 and oe-NC, ${ }^{\$} p<0.05$ compared with cells treated with si-NOTCH2 and oe-NC. The measurement data were expressed as mean \pm standard deviation. The unpaired $t$-test was used to compare the data of the two groups of unpaired designs that followed normal distribution and homogeneity of variance. Data among multiple groups were analyzed by one-way ANOVA, followed by Tukey's post hoc test. The cell experiment was repeated three times independently. 

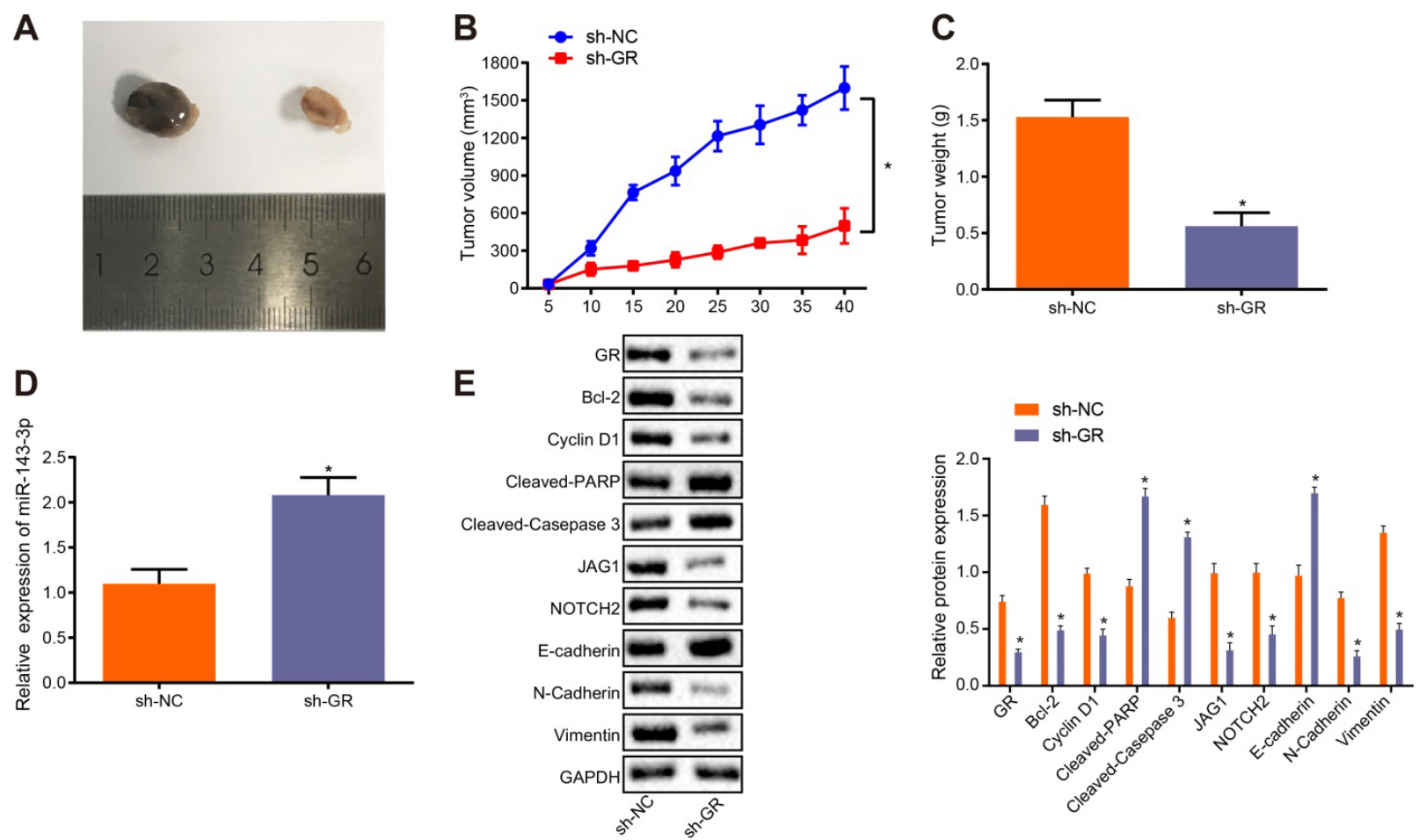

Fig. 6. GR/miR-143-3p/JAG1/NOTCH2 regulates tumorigenesis of CRPC cells in vivo. A, Representative images of tumors formed in nude mice 40 days after injection with CRPC cells. B, The tumor growth curve. C. The final tumor size in nude mice. D, The expression of miR-143-3p in tumor tissues was determined by RT-qPCR. E, The expression of GR, Bcl-2, Cyclin D1, Cleaved-PARP, Cleaved-Caspase 3, JAG1, NOTCH2, E-cadherin and Vimentin normalized to GAPDH in tumor tissues was measured by Western blot analysis. In panel C, D, and E, ${ }^{*} p<0.05$ compared with sh-NC group. All the measurement data were expressed as mean \pm standard deviation. The paired $t$-test $(n=5)$ was used to compare the data of the two groups of paired designs, and the unpaired $t$-test was used to compare the data of the two groups of unpaired designs that followed normal distribution and homogeneity of variance. Data of different groups at different time points were compared by repeated measures ANOVA, and Bonferroni was used for post-test. $N=5$.

\section{Discussion}

As a heterogeneous disease, CRPC has been widely documented as a prostate cancer that no longer responds to ADT, characterized by a series of genetic and epigenetic disorders at a genome level [20]. GR has been identified in literature as being resistant to ADT, which has various effects on CRPC [21]. During the current study, we set out to investigate the effects mediated by the GR gene on CRPC in relation to the involvement of miR-143-3p/JAG1/NOTCH2. Finally, gain- and lossof-function approaches and xenograft tumor model in nude mice were employed in our study, and our results highlighted the significant role of the GR/miR-1433p/JAG1/NOTCH2 axis in CRPC.

Our results demonstrated that GR was highly expressed in CRPC, while miR-143-3p was poorly expressed in CRPC. Previous research has indicated that elevated expression and activity of GR may lead to the development of CRPC following androgen block- ade [9]. Furthermore, it has been previously reported that activation of GR may potentially inhibit tumor growth in preclinical models of prostate cancer with complete androgen receptor signaling, and high expression of GR is related to patients with prostate cancer exposed to ADT [22]. Poorly expressed miR-143-3p has been implicated in multiple types of cancers, including bladder cancer, non-small cell lung cancer, and endometrial carcinoma [23]. Moreover, the down-regulation of miR-143 has been detected in prostate cancer, while the overexpression of miR-143 has been shown to inhibit the proliferation and migration of prostate cancer cells in addition to enhancing their sensitivity to docetaxel [12,24].

Additionally, we obtained evidence illustrating the capacity of GR to down-regulate the expression of miR143-3p, while the down-regulation of GR could curb proliferation, migration and invasion of CRPC cells by up-regulating the expression of miR-143-3p. Glucocorticoid exerts essential functions in metabolism, develop- 


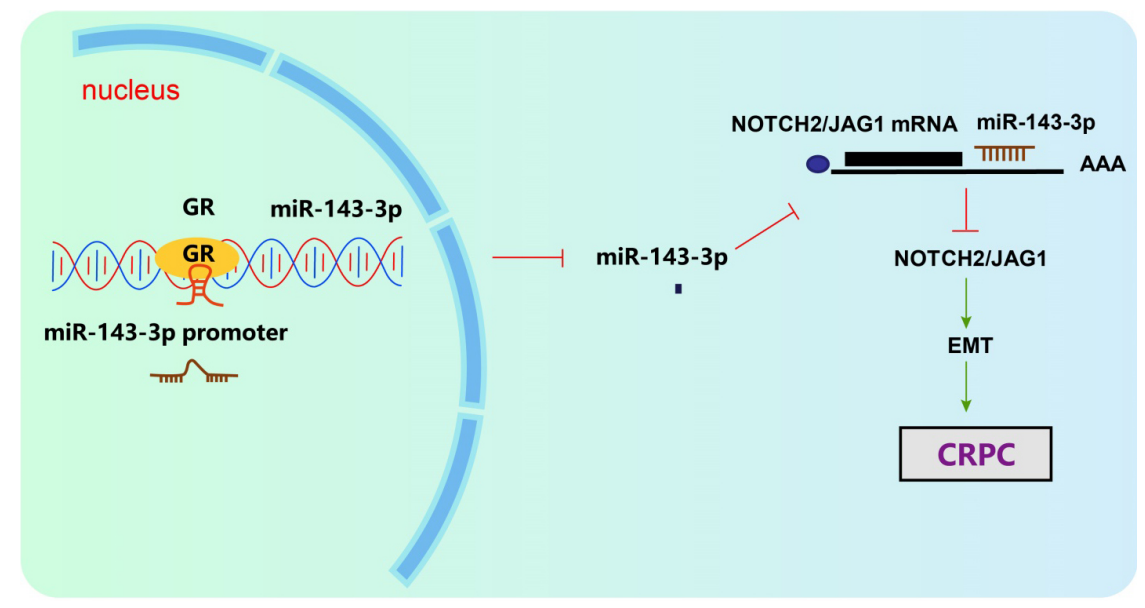

Fig. 7. GR was highly expressed in CRPC, while miR-143-3p was poorly expressed in CRPC. JAG1 and NOTCH2 were the target genes of miR-143-3p. GR could potentially elevate the expression of JAG1/NOTCH2 via inhibition of miR-143-3p. Silencing GR inhibited cell proliferation, migration and invasion through up-regulation of miR-143-3p, thereby suppressing EMT and progression of CRPC.

ment and immune response through GR [25]. Accumulating evidence has noted that glucocorticoid-activated GR can lead to failure of chemotherapy and tumor progression [26]. Moreover, the biology of GR represents a particularly complexity, including ligand-dependent receptor activation and subtype-specific transcription activities, and GR has been reported to modulate not only protein-encoded genes, but also activate non-coding RNA, including miRNA [27]. Previous data have revealed that GR activation stimulates cell proliferation in bladder cancer [28]. Moreover, one previous study has revealed that up-regulated miR-15b and miR-16 in CCRF-CEM T-ALL cells can increase glucocorticoidinduced cell death, highlighting that these miRNAs may be crucial regulators of glucocorticoid-induced apoptosis [29].

Furthermore, we presented evidence implicating that JAG1 and NOTCH2 were highly expressed in CRPC, and JAG1 and NOTCH2 were the target genes of miR-143-3p. Additionally, the GR/miR-143$3 \mathrm{p} / \mathrm{JAG} 1 / \mathrm{NOTCH} 2$ axis has been shown to influence the biological characteristics of CRPC cells by regulating EMT. Previous researchers have verified the role of JAG1/NOTCH2 in EMT that overexpressed JAG1 can promote EMT, while silencing NOTCH2 can reverse the EMT process [30]. Furthermore, miR-143 has been shown to be independently activated by JAG1/NOTCH and SRF in parallel pathways [32]. Decreased miR-143 is observed in the inflammation region of colon, which can also regulate the expression of NOTCH2 and Ecadherin [32]. Partly in line with our results, a previous report concluded effectively suppressed expression of miR-143-3p in metastatic prostate cancer via reverse phase proteome arrays, and it was also reported that reexpression of miR-143-3p can inhibit cell proliferation and target key carcinogenic pathways including cell cycle and apoptosis in prostate cancer [15]. Based on the aforementioned, it is reasonable to believe that GR regulates the expression of JAG1/NOTCH2, the target genes of miR-143-3p, and further regulates the biological characteristics of CRPC cells. Kach et al. have asserted that the activation of GR reduces the anti-tumor effect of AR blockade, and compared with inhibition of AR alone, both the inhibition of GR and AR have been reported to suppress the tumorigenicity of prostate cancer cells in vitro and in vivo [9]. Finally, the in vivo results of our study also demonstrated that the silencing of GR inhibited the proliferation of prostate cancer cells and tumor-forming rate in vivo by up-regulating the expression of miR-143-3p.

In conclusion, GR could inhibit the expression of miR-143-3p, and JAG1 and NOTCH2 were the target genes of miR-143-3p. The central observations of the current study shed light on the mechanism by which GR promoted the progression of CRPC through the JAG1/NOTCH2 pathway by down-regulating miR-143$3 p$ (Fig. 7). Our study proposes a notable role for the GR/miR-143-3p/JAG1/NOTCH2 axis in CRPC. However, we failed to reach a general conclusion since that most experiments were conducted at a cellular level and the data of animal-level experiments were relatively scarce. Hence, further investigations into the current topic are required with a larger sample size in vivo for validation. 


\section{Acknowledgments}

We acknowledge and appreciate our colleagues for their valuable efforts and comments on this paper.

This study was supported by the Yunnan Provincial Health and Family Planning Commission Medical Discipline Leaders Training Program (Grant No. D-201670).

\section{Conflict of interest}

The authors declare no potential conflicts of interest.

\section{References}

[1] G. Attard, C. Parker, R.A. Eeles, F. Schroder, S.A. Tomlins, I Tannock, C.G. Drake and J.S. de Bono, Prostate cancer, Lancet 387 (2016), 70-82.

[2] F. Bray, J. Ferlay, I. Soerjomataram, R.L. Siegel, L.A. Torre and A. Jemal, Global cancer statistics 2018: GLOBOCAN estimates of incidence and mortality worldwide for 36 cancers in 185 countries, CA Cancer J Clin 68 (2018), 394-424.

[3] Y. Huang, X. Jiang, X. Liang and G. Jiang, Molecular and cellular mechanisms of castration resistant prostate cancer, Oncol Lett 15 (2018), 6063-6076.

[4] S.J. Kim and S.I. Kim, Current treatment strategies for castration-resistant prostate cancer, Korean J Urol 52 (2011), 157-165.

[5] T. Chandrasekar, J.C. Yang, A.C. Gao and C.P. Evans, Mechanisms of resistance in castration-resistant prostate cancer (CRPC), Transl Androl Urol 4 (2015), 365-380.

[6] K. Simondsen and J. Kolesar, New treatment options for castration-resistant prostate cancer, Am J Health Syst Pharm 70 (2013), 856-865

[7] T.A. Yap, A. Zivi, A. Omlin and J.S. de Bono, The changing therapeutic landscape of castration-resistant prostate cancer, Nat Rev Clin Oncol 8 (2011), 597-610.

[8] R. Abduljabbar, O.H. Negm, C.F. Lai, D.A. Jerjees, M. AlKaabi, M.R. Hamed, P.J. Tighe, L. Buluwela, A. Mukherjee, A.R. Green, S. Ali, E.A. Rakha and I.O. Ellis, Clinical and biological significance of glucocorticoid receptor (GR) expression in breast cancer, Breast Cancer Res Treat 150 (2015), 335-346.

[9] J. Kach, T.M. Long, P. Selman, E.Y. Tonsing-Carter, M.A. Bacalao, R.R. Lastra, L. de Wet, S. Comiskey, M. Gillard, C. VanOpstall, D.C. West, W.C. Chan, D.V. Griend, S.D. Conzen and R.Z. Szmulewitz, Selective glucocorticoid receptor modulators (SGRMs) delay castrate-resistant prostate cancer growth, Mol Cancer Ther 16 (2017), 1680-1692.

[10] W. Thieu, D. Tilki, R. de Vere White and C.P. Evans, The role of microRNA in castration-resistant prostate cancer, Urol Oncol 32 (2014), 517-523.

[11] C. Xia, Y. Yang, F. Kong, Q. Kong and C. Shan, MiR-143-3p inhibits the proliferation, cell migration and invasion of human breast cancer cells by modulating the expression of MAPK7, Biochimie 147 (2018), 98-104.

[12] B. Xu, X. Niu, X. Zhang, J. Tao, D. Wu, Z. Wang, P. Li, W. Zhang, H. Wu, N. Feng, Z. Wang, L. Hua and X. Wang, miR-
143 decreases prostate cancer cells proliferation and migration and enhances their sensitivity to docetaxel through suppression of KRAS, Mol Cell Biochem 350 (2011), 207-213.

[13] F. Xie, C. Li, X. Zhang, W. Peng and T. Wen, MiR-143-3p suppresses tumorigenesis in pancreatic ductal adenocarcinoma by targeting KRAS, Biomed Pharmacother 119 (2019), 109424.

[14] H. Shi, H. Shen, J. Xu, S. Zhao, S. Yao and N. Jiang, MiR-143 3p suppresses the progression of ovarian cancer, Am J Transl Res 10 (2018), 866-874.

[15] C. Coarfa, W. Fiskus, V.K. Eedunuri, K. Rajapakshe, C. Foley, S.A. Chew, S.S. Shah, C. Geng, J. Shou, J.S. Mohamed, B.W O'Malley and N. Mitsiades, Comprehensive proteomic profiling identifies the androgen receptor axis and other signaling pathways as targets of microRNAs suppressed in metastatic prostate cancer, Oncogene 35 (2016), 2345-2356.

[16] R. O'Brien and L. Marignol, The Notch-1 receptor in prostate tumorigenesis, Cancer Treat Rev 56 (2017), 36-46.

[17] Y. Yu, Y. Zhang, W. Guan, T. Huang, J. Kang, X. Sheng and J. Qi, Androgen receptor promotes the oncogenic function of overexpressed Jagged1 in prostate cancer by enhancing cyclin B1 expression via Akt phosphorylation, Mol Cancer Res 12 (2014), 830-842.

[18] B. You and K.C. Zhang, MicroRNA-144-3p inhibits cell proliferation and promotes apoptosis in castration-resistant prostate cancer by targeting CEP55, Eur Rev Med Pharmacol Sci 22 (2018), 7660-7670

[19] J. Sun, X. Tian, J. Zhang, Y. Huang, X. Lin, L. Chen and S Zhang, Regulation of human glioma cell apoptosis and invasion by miR-152-3p through targeting DNMT1 and regulating NF2: MiR-152-3p regulate glioma cell apoptosis and invasion, J Exp Clin Cancer Res 36 (2017), 100

[20] T. Zhang and A.J. Armstrong, Clinical phenotypes of castration-resistant prostate cancer, Clin Adv Hematol Oncol 11 (2013), 707-718

[21] J. Hu and Q. Chen, The role of glucocorticoid receptor in prostate cancer progression: from bench to bedside, Int Uro Nephrol 49 (2017), 369-380.

[22] M. Isikbay, K. Otto, S. Kregel, J. Kach, Y. Cai, D.J. Vander Griend, S.D. Conzen and R.Z. Szmulewitz, Glucocorticoid receptor activity contributes to resistance to androgen-targeted therapy in prostate cancer, Horm Cancer 5 (2014), 72-89.

[23] L. Guo, J. Fu, S. Sun, M. Zhu, L. Zhang, H. Niu, Z. Chen, Y. Zhang, L. Guo and S. Wang, MicroRNA-143-3p inhibits colorectal cancer metastases by targeting ITGA6 and ASAP3, Cancer Sci 110 (2019), 805-816.

[24] P. Zhou, W.G. Chen and X.W. Li, MicroRNA-143 acts as a tumor suppressor by targeting hexokinase 2 in human prostate cancer, Am J Cancer Res 5 (2015), 2056-2063.

[25] Z. Chen, X. Lan, D. Wu, B. Sunkel, Z. Ye, J. Huang, Z. Liu S.K. Clinton, V.X. Jin and Q. Wang, Ligand-dependent genomic function of glucocorticoid receptor in triple-negative breast cancer, Nat Commun 6 (2015), 8323.

[26] D. Pan, M. Kocherginsky and S.D. Conzen, Activation of the glucocorticoid receptor is associated with poor prognosis in estrogen receptor-negative breast cancer, Cancer Res 71 (2011), 6360-6370.

[27] D. Buschmann, R. Gonzalez, B. Kirchner, C. Mazzone, M.W. Pfaffl, G. Schelling, O. Steinlein and M. Reithmair, Glucocorticoid receptor overexpression slightly shifts microRNA expression patterns in triple-negative breast cancer, Int J Oncol 52 (2018), 1765-1776.

[28] Y. Zheng, K. Izumi, Y. Li, H. Ishiguro and H. Miyamoto, Contrary regulation of bladder cancer cell proliferation and 
invasion by dexamethasone-mediated glucocorticoid receptor signals, Mol Cancer Ther 11 (2012), 2621-2632.

[29] J. Rainer, C. Ploner, S. Jesacher, A. Ploner, M. Eduardoff, M. Mansha, M. Wasim, R. Panzer-Grumayer, Z. Trajanoski, H. Niederegger and R. Kofler, Glucocorticoid-regulated microRNAs and mirtrons in acute lymphoblastic leukemia, Leukemia 23 (2009), 746-752.

[30] J. Chen, H. Zhang, Y. Chen, G. Qiao, W. Jiang, P. Ni, X. Liu and L. Ma, miR-598 inhibits metastasis in colorectal cancer by suppressing JAG1/Notch2 pathway stimulating EMT, Exp Cell Res 352 (2017), 104-112.
[31] J.M. Boucher, S.M. Peterson, S. Urs, C. Zhang and L. Liaw, The miR-143/145 cluster is a novel transcriptional target of Jagged-1/Notch signaling in vascular smooth muscle cells, $J$ Biol Chem 286 (2011), 28312-28321.

[32] E. Boros, M. Csatari, C. Varga, B. Balint and I. Nagy, Specific gene- and MicroRNA-expression pattern contributes to the epithelial to mesenchymal transition in a rat model of experimental colitis, Mediators Inflamm 2017 (2017), 5257378

\section{Supplementary data}
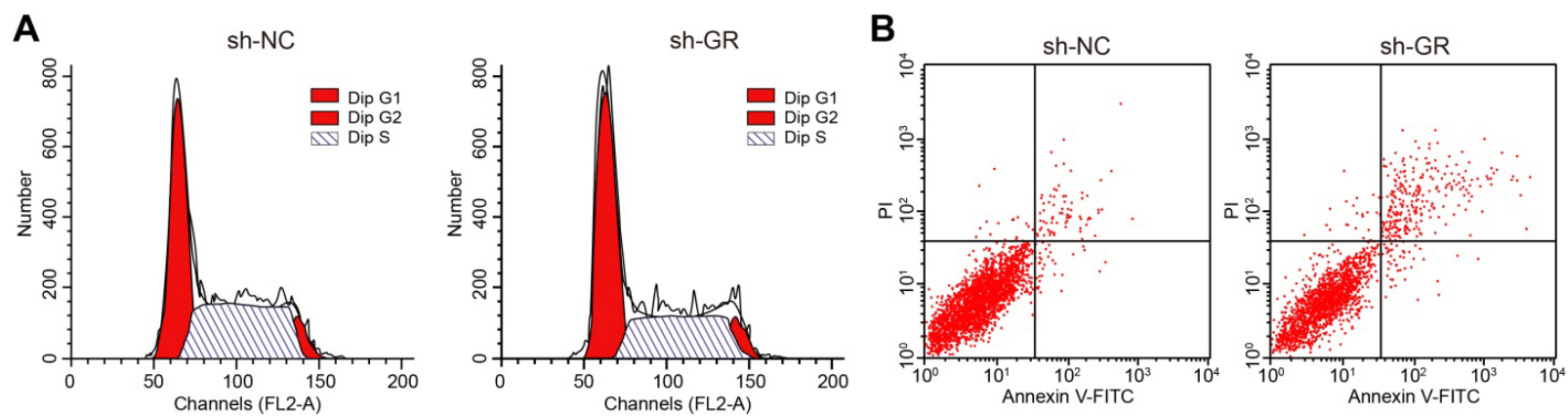

Supplementary Fig. 1. The down-regulation of GR inhibits cell cycle progression and promotes CRPC cell apoptosis. A, Cell cycle distribution analyzed by PI signal staining of flow cytometry. B, Cell apoptosis assessed by Annexin V-FITC/PI dual staining of flow cytometry. 